Time Series Momentum Trading Strategy and Autocorrelation Amplification

K. J. Hong and S. Satchell

June 2013

CWPE 1322 


\title{
Time Series Momentum Trading Strategy and Autocorrelation Amplification
}

\author{
K. J. Hong ${ }^{\mathrm{a}, *}$ and S. Satchell ${ }^{\mathrm{b}}$
}

Current Version: May 23, 2013

${ }^{a}$ University Technology of Sydney, Ultimo Rd, Haymarket NSW 2000, Australia

${ }^{b}$ Trinity College, University of Cambridge, Address: Trinity College, Cambridge, CB2 ITQ, U.K

\begin{abstract}
This article assumes general stationary processes for prices and derives the autocorrelation function for a general Moving Average (MA) trading rule to investigate why this rule is used. The result shows that the MA rule is popular because it can identify price momentum and is a simple way of tracing and exploiting price autocorrelation structure without necessarily knowing its precise structure. We focus on analyzing the impact of price momentum on the profitability of the MA rule because the price momentum effect tends to be stronger and more persistent than the return momentum effect.
\end{abstract}

* Corresponding author. Tel: +61 295144028

E-mail address: hong.jimmy@gmail.com 


\section{Introduction}

While fundamental analysts study a company's underlying indicators of profit such as earnings, dividends, new products and $R \& D$, technical analysts focus mainly on price and return but, unwittingly or otherwise, also take into account psychological aspects in the demand for a company's stock. Technicians employ many techniques, one of which is the use of charts. Using charts, technical analysts seek to identify price patterns and market trends in financial markets and attempt to exploit these patterns. Traders and portfolio managers continue to use technical analysis that forecast stock price movements using historical prices to formulate buy and sell decisions. Momentum is an active research topic and recent studies include Grinblatt and Moskowitz (2003), Choria and Shivakumar (2006), Sadka (2006), Zhu and Zhou (2009), Hou, Karolyi and Kho (2011), Marx (2012), Fama and French (2012), Moskowitz, Ooi and Pedersen. (2012, MOP hereafter), Bajgrowicz and Sxaillet (2012) and Menkhoff, Sarno, Schmeling and Schrimpf (2012). Some earlier studies suggest that technical analysis beats the market in risk neutral terms, hence its popularity.

One of the simplest and most widely used trading strategies based on technical analysis is the Moving Average (MA) rule. It is an objective rule-based trading strategy in which buy and sell signals are determined by the relative magnitudes of short and long term MAs. An objective rule-based trading system has well-defined and indisputable buy and sell signals following a decision rule based on past data. There are a number of published papers on MA rules including Acar and Satchell (1997), Chiarella, He and Hommes (2006) and Menkhoff (2010). The MA rule often leads investors to invest with or against the trend, or momentum since it assumes that prices trend directionally. It takes advantage of price trend, which is closely linked with the rather nebulous idea of price momentum. (Price) Momentum is defined by existing literatures as the product of the relative prices over the holding period and a past winners strategy is to buy the top n-th of stocks with this characteristic in a portfolio. A careful reading of the momentum literatures fails to find clarification over whether momentum is present when the price of the asset follows (a) a random walk (b) a random walk with drift or (c) a mean reverting process around a linear trend. The issue is further complicated by different treatments for prices, returns and earnings. One of the purposes of this paper is to try and understand the time series basis of a momentum strategy.

Unlike other momentum literatures that focus on the relative performance of securities in the cross-section, we emphasize that this paper intends to investigate time series 
momentum, the momentum within an asset price process. MOP also look at time series momentum and argue that time series momentum directly matches the predictions of many prominent behavioral and rational asset pricing theories. Barberis, Shleifer and Vishny (1998), Daniel, Hirshleifer and Subrahmanyam (1998), and Hong and Stein (1999) all focus on a single risky asset, therefore having direct implications for time series, rather than crosssectional, predictability. Like-wise, rational theories of momentum (Berk, Green and Naik 1999; Johnson, 2002; Ahn, Conrad and Dittmar, 2003; Sagi and Seasholes, 2007; Liu and Zhang, 2008) also pertain to a single risky asset. Time series momentum focuses purely on a security’s own past process. MOP investigate 58 liquid instruments of equity index, currency, commodity, and bond futures and find it exists in all assets.

We assume general stationary processes for prices and derive the autocorrelation function for a general MA rule. We focus on analysing the impact of price momentum, instead of returns or earnings momentum, on the profitability of the MA rule because (1) most of, if not all, trading rules using technical analysis are based on past prices, and (2) the price momentum effect tends to be stronger and longer-lived than the return momentum effect. There exists another type of price trend besides the time series and cross-section momentum discussed, however. Equity prices tend to show a long run market-wide trend. This can be due to inflation or other economic conditions. We define this market-wide trend by the parameter, $\mu$. We remove the market-wide trend from our price process in order to focus on price momentum only.

This paper presents a simple and straightforward evaluation of the MA rule with one investor and one asset. We find that that there are two reasons why the MA rule is popular. First, the MA rule can identify the price momentum (trend), which comfortably confirms the results of the previous momentum literatures. Second, the MA rule is a simple way of tracing and exploiting the price autocorrelation structure without actually knowing its structure. It is very hard, if not impossible, to know the real structure of the price autocorrelation of an asset. The MA rule provides a simple and clear methodology that can take advantage of the price autocorrelation structure. The main contribution of this paper is that we suggest two reasons why the technical trading strategy, or Chartism, is popular and this leads to price autocorrelation.

Our finding of the autocorrelation structure of the trading rule used in this study directly matches the findings of many prominent behavioral and rational asset pricing theories. For example, if a conservatism bias exists in a market, the MA rule would be 
profitable. A conservatism bias indicates that investors are too slow (too conservative) in updating their beliefs in response to recent evidence. This means that they might initially underreact to news about a firm, meaning that prices will fully reflect new information only gradually. Such a bias would give rise to momentum (price autocorrelation) in the stock market. Using AR (1) and MA (1) processes, we take the discussion away from short term price trend continuation or long term price reversal. In short, it is not just about buying past losers or winners but is also about price trends and the autocorrelation structure. This paper does not intend to explain all the reasons for predictability nor does it attempt to explain why arbitrage fails to eliminate mispricing.

The contribution of the paper is clear.

1. Unlike previous literatures that mainly investigate cross sectional momentum among various equities, this paper investigates time series momentum. The result suggests that momentum trading strategy is popular not only because it takes advantage of trend within the times series data but also because it amplifies the autocorrelation. This shows that a time series momentum trading strategy is a simple way of conditioning on past price information.

2. The paper further investigates the structural change of autocorrelation to identify the pattern of the amplification. This is important because such increase in autocorrelation is not captured by the standard risk measures such as VaR. The paper provides a guideline of how much amplification is occurring. In this respect, the result of the paper has risk management policy implication.

3. The paper also provides theoretical justification to wide use of longer period MA for price momentum and shorter period MA for return momentum.

This paper could be seen as an extension of MOP in that it investigates another aspect of time series momentum, its autocorrelation structure. The current study therefore expands our knowledge of time series momentum. The result has risk management and regulatory policy implications. It reports un-captured potential source of risk in one of the most popular trading strategies. 
The rest of the paper is organized as follows. Section 2 explains times series momentum, the trading rule and how it is related to price momentum. Section 3 presents the general model. Section 4 shows the autocorrelation structure of the trading strategy. Section 5 presents empirical evidence and Section 6 concludes the paper.

\section{Backgrounds}

\subsection{Time Series Price Momentum and Technical Trading Rule}

Empirical studies of technical analysis, including Brock, Lakonishok and LeBaron (1992), Blume, Easley and O’Hara (1994), Chan, Jegadeesh and Lakonishock (1996), Brown, Goetzmann and Kumar (1998), Allen and Karjalainen (1999), Chan, Hameed and Tong (2000), Lo, Mamaysky and Wang (2000) and Hou, Karolyi and Kho (2011) focus on the relative performance of securities in the cross-section. Many literatures find that such cross sectional model works very well. However, Lewellen, Nagel and Shanken (2010) argue that the empirical methodologies used in cross sectional asset pricing literatures are often highly misleading, in the sense that apparently strong explanatory power (high cross-sectional $\mathrm{R}^{2} \mathrm{~s}$ and small pricing errors) can provide quite weak support for a model. There is another kind of momentum that could be investigated, time series momentum. MOP argue, "Time series momentum is the momentum within asset price process. It is related to but different from the phenomenon known as 'momentum' in the finance literature, which is primarily crosssectional in nature. The momentum literature focuses on the relative performance of securities in the cross-section, finding that securities that recently outperformed their peers over the past three to 12 months continue to outperform their peers on average over the next month. Rather than focus on the relative returns of securities in the cross-section, time series momentum focuses purely on a security’s own past return.”

In this paper, we investigate a technical trading strategy based on this time series momentum with the focus on the autocorrelation structure of the trading strategy. Technical trading rule takes advantage of Time Series Momentum. In support of MOP, we also find that our result is consistent with initial under-reaction and delayed over-reaction stories. We employ and expand the definition of the MA oscillator by Brock, Lakonishock and LeBaron (1992), which states: “According to the moving average trading rule, buy and sell signals are 
generated by two moving averages of the level of the index - a long-period average and a short-period average. In its simplest form this strategy is expressed as buying (or selling) when the short-period moving average rises above (or falls below) the long-period moving average.

The idea behind computing moving averages it to smooth out an otherwise volatile series. When the short-period average exceeds the long-period moving average, a trend is considered to be initiated. A very popular moving average rule is $1-200$, where the short period is one day and the long period is 200 days. While numerous variations of this rule are used in practice, we attempted to select several of the most popular ones: 1-50, 1-150, 5-150, 1-200, and 2-200.” However, we can also take into consideration the flip side of the strategy, where we buy when the short-period MA falls below the long-period MA and we sell when the opposite happens.

Let $l$ stand for the time period that the MA is computed for a long position and $s$ for the time period that the MA is computed for a short position. Hence $s>l$ and they overlap. Denote SMA as MA computed over time period $s$ and LMA as MA computed over time period $l$. For example at time t, if $s=7$ and $l=3$, then SMA is computed over time $\mathrm{t}-6$ to $\mathrm{t}$ and LMA is computed over time $\mathrm{t}-2$ to $\mathrm{t}$. We classify two opposite MA rules with SMA and LMA, MAbull $(l, s)$ and MAbear $(l, s)$, and define the MAbull $(l, s)$ rule as taking a long position in the asset when SMA > LMA and as taking no position in the asset when SMA < LMA and MAbear $(l, s)$ rule does the opposite.

Definition 1: The MAbull(l,s) and MAbear $(l, s)$ position can be represented as

$$
\operatorname{MAbull}(l, s)=\max (\delta(t), 0) \quad \text { MAbear }(l, s)=\max (1-\delta(t), 0)
$$

where $\delta(t)=\left\{\begin{array}{l}1 \text { if } L M A(t)-S M A(t)>1 \\ 0 \text { if } L M A(t)-S M A(t)<1\end{array}\right.$

and $\operatorname{SMA}(t)=\frac{\sum_{i=1}^{s} P(t-i+1)}{s}$ and $\operatorname{LMA}(t)=\frac{\sum_{i=1}^{l} P(t-i+1)}{l}$

The logic behind the MAbull rule is well known, when a price penetrates the MA from below, the bull trend is believed to be established and a trader wants to take advantage of this 
in the expectation that there will be further upward movements in prices. The MAbear rule tells us the opposite story, known to practitioners as short term price reversal. When the price falls enough to penetrate the MA from above, this might be due to market over-reaction; hence a trader would take a long position, expecting that the price would bounce immediately back.

The relationship between the MA rule and price momentum is well explained in Fong and Yong (2005). "In the simplest form, a MA crossover rule operates on the assumption that buy signals are generated when the current stock price crosses its moving average from below while sell signals are generated when the stock price crosses its moving average from above. The rationale for this interpretation is that a trend is said to have emerged when the stock price penetrates the moving average. Specifically, an upward (bullish) trend emerges when the price rises above its moving average, while a downward (bearish) trend emerges when the price falls below its moving average." Therefore, the MAbull rule takes advantage of the bullish trend while the MAbear rule takes advantage of the bearish trend. MAbull strategy can be thought as buying a past loser because it takes a long position when the asset price is relatively low and liquidates the position when the asset price is relatively high.

\subsection{Momentum Definition and a Statistical Model}

Hong and Satchell (2012) consider the process

$$
d q(t)=-\theta q(t) d t+\sigma d W(t)
$$

where $q(t):=\log P(t)-\mu t$

With the Ornstein Uhlenbeck process above, they investigate the definition of momentum proposed by Achelis, momentum can be interpreted as the sensitivity of the expected price ratio with respect to today's price. If this sensitivity is positive then there is a bullish momentum and if negative there is a bearish momentum. Proposition 1 of Hong and Satchell (2012) shows the log conditional population momentum with respect to pervious period's log price. They note that for log OU, momentum is about autocorrelation. Equation (3) shows that when the price process is mean reverting, the elasticity of momentum is negative while it is positive if the price is explosive. This is exactly what we would anticipate. In other words, 
given the high past price, the future price is low if the price process reverts around a mean but the future price is high if the price process is explosive. If we were to interpret efficient markets as a log random walk with drift, then the elasticity would become zero. Finally, the elasticity is independent of the trend.

\subsection{Clarification of Notation}

We employ a great deal of notations in this paper. Some only differ in super and sub scripts, some are context specific. For example, $\Omega$ with or without super and subscripts stand for a covariance matrix. $\Omega$ stands for the covariance matrix of current de-meaned price process, $\Omega_{m}$ stands for the covariance matrix between the current and m-period lag de-meaned price process and $\Omega^{T}$ indicates the covariance matrix of the trading strategy critical value. We include a glossary in Appendix A1 to help readers.

\section{The Model}

\subsection{General Model}

Let the de-meaned $\log$ price process $q(t)=\log P(t)-\mu t$ has distribution $q(t) \sim\left(0, \sigma^{2}\right)$. Also denote the autocorrelation between $q(t)$ and $q(t-h)$ as $\rho_{A}(h)$. Since we are using log prices, it would be sensible to use geometric price MAs which are equivalent to arithmetic log price MAs.

$$
\begin{gathered}
\operatorname{SMA}(t)=\exp \left(\frac{\sum_{i=1}^{s} q(t-i+1)}{s}\right) \text { and } L M A(t)=\exp \left(\frac{\sum_{i=1}^{l} q(t-i+1)}{l}\right) \\
\text { where } s>l
\end{gathered}
$$

Under such a framework, the return at time $\mathrm{t}$ is defined as

$$
q(t)-q(t-1)=\log P(t)-\log P(t-1)-\mu
$$


Definition 2: The critical value of a $M A(s, l))$ rule is defined as

$$
C(s, l ; t)=\frac{L M A(t)}{S M A(t)}=\exp \left(w^{\prime} Q(t)\right)
$$

$$
\begin{aligned}
& \text { where } w^{\prime}=\left(\begin{array}{lll}
\left(\frac{1}{l}-\frac{1}{s}\right) & \cdots & \left(\frac{1}{l}-\frac{1}{s}\right)
\end{array}-\left(\frac{1}{s}\right) \cdots-\left(\frac{1}{s}\right)\right) \text { and } \\
& Q(t)^{\prime}=\left(\begin{array}{llllll}
q(t) & q(t-1) & \cdots & q(t-l+1) & \cdots & q(t-s+1)
\end{array}\right)
\end{aligned}
$$

The derivation of definition 2 is in Appendix A2. Take a long position if $\mathrm{C}(s, l ; \mathrm{t})$ becomes more than 1 and liquidate the position if $\mathrm{C}(s, l ; \mathrm{t})$ becomes less than 1 . When the MA rule is applied based on a positive trending price process, the exit point (where the manager liquidates a long position due to the current price penetrating the MA from above) will always be higher than the entry point hence the return is always positive. However, the manager is simply picking up the price trend and the same return can also be made with a buy and hold strategy. This is less interesting. The importance of the autocorrelation within a financial time series is also noted in MOP, as they state "We find that positive autocovariance in our futures contracts' return drives most of the time series and cross-sectional momentum effects we find in the data. The contribution of the other two return components serial cross-correlations and variation in mean returns - is small”. In order to separate the impact of momentum from the market-wide trend, we use the detrended log price, which also has useful statistical properties for estimation. The 'detrended' MA rule also has a simple price interpretation as shown below. Since MAbear rule is simply the opposite of the MAbull rule, we only look at the MAbull hereafter. However the MAbear rule would be useful as one of benchmarks in evaluating the profitability of MAbull rule.

Assuming stationarity in the second moments, the bivariate distribution of $\mathrm{C}(\mathrm{t})$ and $\mathrm{C}(\mathrm{t}-1)$ can be expressed as,

$$
\left(\begin{array}{c}
w^{\prime} Q(t) \\
w^{\prime} Q(t-m)
\end{array}\right) \sim N\left(\left(\begin{array}{l}
0 \\
0
\end{array}\right)\left(\begin{array}{cc}
w^{\prime} \Omega w & w^{\prime} \Omega_{m} w \\
w^{\prime} \Omega_{m} w & w^{\prime} \Omega w
\end{array}\right)\right)
$$


where $\Omega$ is the $(s \times s)$ covariance matrix of $\mathrm{Q}(\mathrm{t})$ and $\Omega_{C}$ is $(s \times s)$ covariance matrix between $\mathrm{Q}(\mathrm{t})$ and $\mathrm{Q}(\mathrm{t}-1)$. For now, we do not assume anything about the covariance matrix but simply note that the autocovariance is not zero.

Lemma 1: The MAbull $(l, s)$ rule based on a detrended log price is equivalent to buying a unit of an asset when

$$
P(t)>\exp \left(\frac{\mu m}{2}\right)\left(\prod_{j=1}^{m-1} P(t-j)\right)^{\frac{1}{m-1}}
$$

The derivation of Lemma 1 is in Appendix A3. Under our log Ornstein Uhlenbeck assumptions, $\mathrm{C}(s, l ; \mathrm{t})$ has a log normal distribution.

$$
C(s, l ; t)=\exp \left(w^{\prime} Q(t)\right) \quad w^{\prime} Q(t) \sim N\left(0, w^{\prime} \Omega w\right)
$$

Although we apply this model to the price process only, the application of the model does not need to be restricted to the price process only. It can also be applied to returns or earnings or to any process that would have time series momentum (autocorrelation / trend). This point will be discussed again later in the paper.

\subsection{The Profitability of the MA Rule}

Before we discuss the profitability, we have to emphasize that this paper does not intend to investigate the profitability of the MA rule. The only reason that we discuss the profitability is because it helps to investigate the transition vector of the critical value, $\mathrm{C}(s, l ; \mathrm{t})$ distribution. However we do not wish to get into a full length discussion of the profitability of the trading rule, which is not the main topic of interest in this paper. We plan to investigate this topic in a separate study. This point will be emphasized again when we perform empirical investigations.

Definition 3: Define $\alpha(s, l ; t)$ as 


$$
\alpha(s, l ; t)=\left\{\begin{array}{l}
1 \text { if } \mathrm{C}(s, l ; t)>1 \Leftrightarrow w^{\prime} Q(t)>0 \\
0 \text { if } \mathrm{C}(s, l ; t)<1 \Leftrightarrow w^{\prime} Q(t)<0
\end{array}\right.
$$

hence $E[\alpha(s, l ; t)]=\operatorname{prob}(C(s, l ; t)>1)$

Remark 1: The unconditional and conditional probability of $\alpha(s, l ; t)$ taking the value of 1 under normality in prices can be expressed as

$$
\text { Unconditional: } \operatorname{prob}(\alpha(s, l ; t)=1)=0.5
$$

$$
\text { Conditional: } \operatorname{prob}(\alpha(s, l ; t)=1 \mid \xi)=1-\Phi\left(\frac{-\rho_{T}(1)\left(q_{t-1}-S M A(t-1)\right)}{\sqrt{\left(1-\rho_{T}^{2}(1)\right) w^{\prime} \Omega w}}\right)
$$

where $\rho_{T}(1)$ : the first order of the trading strategy autocorrelation and $\xi=w^{\prime} Q(t-1)=q_{t-1}-S M A(t-1)$.

The proof of Remark 1 is in Appendix A4.

Proposition 2: The $h$ period return from the MAbull rule from time $k, r_{T}(k+h, k)$ can be expressed as

$$
r_{T}(s, l ; k+h, k)=\sum_{i=1}^{h}\left(\alpha(s, l ; k+i-1 \mid k) \times r_{A}(k+i, k+i-1)\right)
$$

where $r_{T}(s, l ; k+h, k)$ is the $h$ period return from the MAbull rule from time $k$ to time $k+h$, given $\mathrm{s}$ and l. $r_{A}(k+h, k)$ is the $\mathrm{h}$ period asset return from time $k$ to time $k+h$. If $r_{T}(k+h, k)>r(k+h, k)$, then the MA rule is profitable. This is only possible if $\alpha$ is more likely to take a value of 1 when the asset return is positive. This provides with us a condition that would make the MAbull rule profitable. If $\rho_{T}(1)>0$ and $\xi>0$, the conditional probability of $\alpha$ being equal to one larger than 0.5 when $q_{t}>q_{t-1}$, hence the MAbull rule is profitable. 
Analytically analyzing equation (8) is too complicated hence simulation would be useful. We noted that price autocorrelation is important in determining the conditional probability of $\alpha(s, l ; t)$. Thus an assumption regarding price autocorrelation needs to be made and we assume that the asset price follows the AR(1) process. We use Goldman Sachs (GS) data from May 4, 1999 to May 31, 2011 for estimation. There is no special reason for selecting such data and time period. The estimation is only to locate some sensible parameters. The simulation process is summarized in Appendix A5. This paper is concerned with price (level), which tends to have very high autocorrelation. Hence an investigation of high level autocorrelations would be more meaningful. For convenience, we examine the MA(1,10) rule.

Table 1: Simulated profitability of the MA(1,10) rule when asset price follows an AR(1) process for $\rho=0.99,0.925,0.995,0.9975$ and under normality

\begin{tabular}{lrrrr} 
Annual Profit & & & & \\
\hline Rho & 0.9900 & 0.9925 & 0.9950 & 0.9975 \\
MA bull & $13.19 \%$ & $13.45 \%$ & $13.94 \%$ & $15.09 \%$ \\
MA bear & $-15.54 \%$ & $-15.01 \%$ & $-13.92 \%$ & $-11.73 \%$ \\
Buy and Hold & $1.79 \%$ & $2.40 \%$ & $3.55 \%$ & $6.54 \%$ \\
\hline
\end{tabular}

Table 1 presents the simulated results. The error terms are simulated to have a normal distribution. The simulated result shows that the conditioning on previous price information affects the profitability of the trading strategy when there is positive price autocorrelation. The MAbull rule outperforms the other strategies because there is a positive trend in the simulated $\log$ price process hence $\operatorname{prob}(\alpha(s, l ; t)=1 \mid \xi)>0.5$ when the asset return is positive. This confirms the results of previous literature including Fong and Yong (2005) and is what we exactly anticipated. The result also highlights that the degree of autocorrelation affects the performance of the trading strategy as well.

The portfolio position is determined by the sign of $C(s, l ; t)$, therefore a further investigation of $C(s, l ; t)$ transition with respect to time would be meaningful. To gain insight into the properties of the $\operatorname{MA}(1, s)$ rule, we calculate a transition matrix for $C(s, l ; t)$. This calculates different values for the autocorrelation probability of $C(s, l ; t)$ that is how it changes from one sign to the other. If the price process was random and symmetric, this would be a quarter in all regions. 
Table 2: C(s,l;t) Transition Vector of the Simulated Results of the MAbull $(1,10)$ rule

\begin{tabular}{lrrrr}
\hline & \multicolumn{1}{c}{0.99} & \multicolumn{1}{c}{0.9925} & \multicolumn{1}{c}{0.995} & \multicolumn{1}{c}{0.9975} \\
\hline R1(+,+) & $44.44 \%$ & $45.25 \%$ & $49.60 \%$ & $54.04 \%$ \\
R2(+,-) & $7.68 \%$ & $8.89 \%$ & $9.49 \%$ & $7.37 \%$ \\
R3(-,+) & $7.58 \%$ & $8.99 \%$ & $9.60 \%$ & $7.47 \%$ \\
R4(-,-) & $40.30 \%$ & $36.87 \%$ & $31.31 \%$ & $31.11 \%$ \\
\hline
\end{tabular}

* R1: $\mathrm{C}(\mathrm{t}-1)>0$ and $\mathrm{C}(\mathrm{t})>0 \quad \mathrm{R} 2: \mathrm{C}(\mathrm{t}-1)>0$ and $\mathrm{C}(\mathrm{t})<0$

R3: $\mathrm{C}(\mathrm{t}-1)<0$ and $\mathrm{C}(\mathrm{t})>0 \quad \mathrm{R} 4: \mathrm{C}(\mathrm{t}-1)<0$ and $\mathrm{C}(\mathrm{t})<0$

The probabilities of region 2 and 3 should be symmetric by the construction of the MA rule. When $C(s, l ; t)$ is in Region 1, the MAbull rule is likely to be profitable while when it is in Region 4, the MAbear rule is likely to be profitable. When in Region 2 (3), the MAbull rule will take (liquidate) a position in an asset and the MAbear rule will liquidate (take) a position in an asset. With a positive trend and autocorrelation in price, we see that the asset is more likely to be in Region 1 than in Region 4, which explains why the MAbull rule makes the most profit. The probability of Region 1 increases as price autocorrelation increases, which also corresponds to what we have stated previously. The larger the magnitude of the positive autocorrelation, the longer the positive trend will remain, hence both the MA (both bull and bear) rules should be more profitable. This is also shown in Table 2.

\section{Autocorrelation}

From the model, more specifically from equation (7), and the simulated results we have seen that price autocorrelation is an important factor in the profitability of the MA rule. Hence this section investigates autocorrelation of the trading strategy.

\subsection{General Autocorrelation Structure}

Let $\rho_{A}(k)$ be the asset price autocorrelation in log prices between $\mathrm{k}$ periods. Then the covariance matrix between $\mathrm{Q}(t)$ and $\mathrm{Q}(t-m), \Omega_{m}$, can be expressed as 


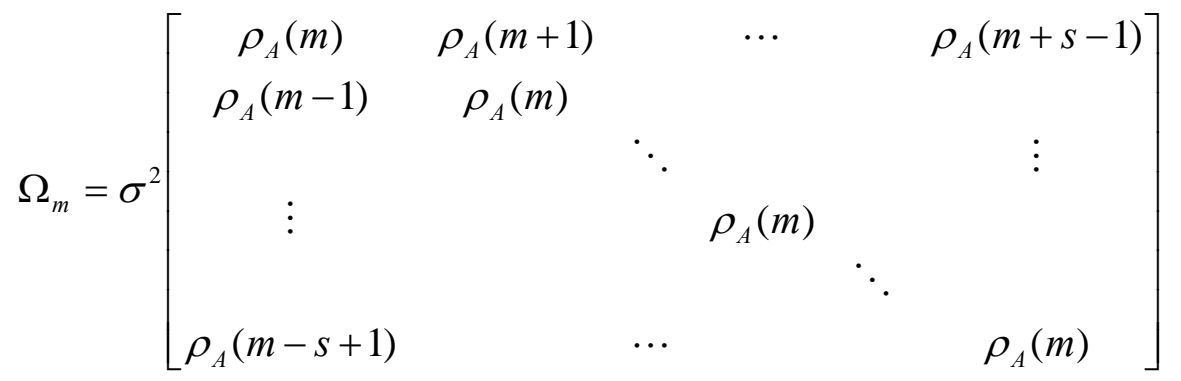

$\rho_{A}(0)=1$ and $\rho_{A}(k)=\rho_{A}(-k)$ since we assume stationarity in second moments. Note that $\Omega$ is a covariance matrix of $\mathrm{Q}(t)$, which is a vector of $\mathrm{q}(i)$ values $(i=1 \ldots t) . \Omega_{m}$ is a covariance matrix between $\mathrm{Q}(t)$ and $\mathrm{Q}(t-1)$.

And the covariance matrix between $C(t)$ and $C(t-m), \Omega_{m}^{T}$, can be expressed as

$$
\Omega_{m}^{T}=\left(\begin{array}{cc}
w^{\prime} \Omega w & w^{\prime} \Omega_{m} w \\
w^{\prime} \Omega_{m} w & w^{\prime} \Omega w
\end{array}\right)
$$

Proposition 3: The variance for $C(s, l ; t)$ and the covariance between $C(s, l ; t)$ and $C(s, l ; t-m)$ can be expressed as

$$
\left.\begin{array}{l}
\Omega_{m}^{T}(1,2)=w^{\prime} \Omega_{m} w= \\
\sigma^{2}\left(l \rho_{A}(m)+\sum_{i=1}^{l-1}\left(\rho_{A}(m+i)+\rho_{A}(m-i)\right)\right) \\
+b^{2}\left((s-l) \rho_{A}(m)+\sum_{i=1}^{s-l-1}(s-i-1)\left(\rho_{A}(m+i)+\rho_{A}(m-i)\right)\right) \\
+a b\left(\sum_{j=0}^{l-1} \sum_{i=0}^{s-l-1} \rho_{A}(m+l+i-j)\right)+a b\left(\sum_{j=0}^{l-1} \sum_{i=0}^{s-l-1} \rho_{A}(m-l-i+j)\right)
\end{array}\right)
$$

where $a=\left(\frac{1}{l}-\frac{1}{s}\right)$ and $b=-\left(\frac{1}{s}\right)$

Proof of Proposition 3 is in Appendix A6. When the MA rule is practically applied, managers often take the shorter time period as $1(l=1)$. When $l=1$, it is referred to as the "MA crossover rule". This case can be treated as a special case since it makes the equation significantly simpler by getting rid of the first of both double summation terms. 
Remark 2: The m-th order autocorrelation between $C(t)=w^{\prime} Q(t)$ and $C(t-m)=w^{\prime} Q(t-m)$, when $l=1, \rho_{T}(1, s, m)$, can be expressed as

$$
\begin{aligned}
& \rho_{T}(1, s, m)=\frac{\Omega_{m}^{T}(1,2)}{\Omega_{m}^{T}(1,1)}=\frac{w^{\prime} \Omega_{m} w}{w^{\prime} \Omega w}= \\
& \left(\begin{array}{l}
\left.a^{2} \rho_{A}(m)+b^{2}\left((s-1) \rho_{A}(m)+\sum_{i=1}^{s-2}(s-i-1)\left(\rho_{A}(m+i)+\rho_{A}(m-i)\right)\right)\right) \\
+a b\left(\sum_{i=0}^{s-2} \rho_{A}(m+i+1)+\sum_{i=0}^{s-2} \rho_{A}(m-i-1)\right)
\end{array}\right) \\
& \left(a^{2}+b^{2}\left((s-1)+\sum_{i=1}^{s-2}(s-i-1)\left(\rho_{A}(i)+\rho_{A}(-i)\right)\right)+a b\left(\sum_{i=0}^{s-2} \rho_{A}(i+1)+\sum_{i=0}^{s-2} \rho_{A}(-i-1)\right)\right)
\end{aligned}
$$

The proof of Remark 2 is omitted. Calculation check of Remark 2 is performed with simulated result and is included in Appendix 7.

Choosing a particular $\mathrm{MA}(l, s)$ rule depends upon the autocorrelation properties of prices. When $s$ is fixed, $w^{\prime} \Omega w$ is a constant. Therefore for $s>l$, the sensitivity of trading strategy autocorrelation with respect to $\mathrm{m}$ is

$$
\frac{\partial \rho_{T}(m)}{\partial m}=\frac{\partial w^{\prime} \Omega_{m}^{T} w}{\partial m}=\left(\begin{array}{l}
\left.\left(a^{2}+b^{2}(s-1)\right) \frac{\partial \rho_{A}(m)}{\partial m}+b^{2} \sum_{i=1}^{s-2}(s-i-1)\left(\frac{\partial \rho_{A}(m+i)}{\partial m}+\frac{\partial \rho_{A}(m-i)}{\partial m}\right)\right) \\
+a b\left(\sum_{i=0}^{s-2} \frac{\partial \rho_{A}(m+i+1)}{\partial m}+\frac{\partial \rho_{A}(m-i-1)}{\partial m}\right)
\end{array}\right)
$$

If $\frac{\partial \rho_{A}(m)}{\partial m}<0$, we have $\frac{\partial \rho_{T}(m)}{\partial m}<0$

It may well be reasonable to design optimal properties. We do not pursue this further but instead look at the links between the Autocorrelation Function (ACF) of the MA rule and some simple time series models.

In the next two sections, the autocorrelation structure of the $1-10$ strategy is investigated. Equation (9) is computed $\mathrm{m}=30$ and $\rho_{A}$ incrementing from 0.05 to 0.95 with an interval of 0.05 . 


\subsection{Investigation with an AR(1) Price Process}

This section investigates the linkage between technical trading rule and the stochastic process. Assume that the de-meaned price $q$ follows an AR(1) process.

$$
q(t)=\rho_{A}(1) q(t-1)+\varepsilon(t) \quad(11) \quad \varepsilon \sim N(0,1)
$$

Then the expected value, variance, m-period autocovariance and autocorrelation of this process is well known.

$$
\begin{gathered}
E[q(t)]=0, \operatorname{Var}[q(t)]=\sigma^{2}=\frac{1}{1-\rho_{A}^{2}(1)} \\
\operatorname{Cov}[q(t), q(t-m)]=\frac{\rho_{A}^{|m|}(1)}{1-\rho_{A}^{2}(1)}, \rho_{A}(m)=\rho_{A}^{|m|}(1)
\end{gathered}
$$

Note that $\sigma^{2}$ stands for the variance of $q(t)$ and $\rho_{A}(m)$ stands for the m-period autocorrelation. The assumed error term is distributed with a variance of 1 . The covariance matrix between $\mathrm{Q}(t)$ and $\mathrm{Q}(t-m), \Omega_{m}$, can be expressed as

$$
\Omega_{m}=\sigma^{2}\left[\begin{array}{cccccc}
\rho_{A}^{|m|}(1) & \rho_{A}^{|m+1|}(1) & & \cdots & & \rho_{A}^{|m+s-1|}(1) \\
\rho_{A}^{|m-1|}(1) & \rho_{A}^{|m|}(1) & & & & \\
& & \ddots & & & \vdots \\
\vdots & & & \rho_{A}^{|m|}(1) & & \\
& & & & \ddots & \\
\rho_{A}^{|m-s+1|}(1) & & \ldots & & & \rho_{A}^{|m|}(1)
\end{array}\right]
$$

Using this result and Remark 2, we can derive the m-th order autocorrelation between the critical values.

Remark 3: The m-th order autocorrelation between $C(t)=w^{\prime} Q(t)$ and $C(t-m)=w^{\prime} Q(t-m)$, when $l=1$ and $q(t)$ follows Equation (11), $\rho_{T}(1, s, m)$, can be expressed as 


$$
\begin{aligned}
& \rho_{T}(1, s, m)= \\
& \left(\begin{array}{l}
a^{2} \rho_{A}^{|m|}(1)+b^{2}\left((s-1) \rho_{A}^{|m|}(1)+\sum_{i=1}^{s-2}(s-i-1)\left(\rho_{A}^{|m+i|}(1)+\rho_{A}^{|m-i|}(1)\right)\right) \\
+a b \sum_{i=0}^{s-2}\left(\rho_{A}^{|m+i+1|}(1)+\rho_{A}^{|m-i-1|}(1)\right)
\end{array}\right) \\
& \left(a^{2}+b^{2}\left((s-1)+\sum_{i=1}^{s-2}(s-i-1)\left(\rho_{A}^{|i|}(1)+\rho_{A}^{|-i|}(1)\right)\right)+a b \sum_{i=0}^{s-2}\left(\rho_{A}^{|i+1|}(1)+\rho_{A}^{|-i-1|}(1)\right)\right)
\end{aligned}
$$

where $a=\left(1-\frac{1}{s}\right)$ and $b=-\left(\frac{1}{s}\right)$

The proof of Remark 2 is omitted. This is a direct result of Remark 2. Comparing equation (12) with $\rho_{A}(m)$ gives us exact magnitude of autocorrelation amplification under the AR(1) price process framework.

Proposition 4: The difference between $m$-th order MA trading strategy $(l=1)$ autocorrelation and $m$-the order de-meaned asset price autocorrelation can be expressed as

$$
\begin{aligned}
& \rho_{T}(1, s, m)-\rho_{A}(m)= \\
& \rho_{A}^{|m|}(1) b^{2}\left((s-2) \sum_{i=1}^{s-2}(s-i-1)\left(\rho_{A}^{|i|}(1)+\rho_{A}^{|-i|}(1)\right)-\sum_{i=1}^{s-2}\left(\rho_{A}^{|i|}(1)+\rho_{A}^{|-i|}(1)\right)\right) \\
& \quad+a b(s-3) \rho_{A}^{|m|}(1)\left(\sum_{i=0}^{s-2} \rho_{A}^{|i+1|}(1)+\sum_{i=0}^{s-2} \rho_{A}^{|-i-1|}(1)\right) \\
& \left(a^{2}+b^{2}\left((s-1)+\sum_{i=1}^{s-2}(s-i-1)\left(\rho_{A}^{|i|}(1)+\rho_{A}^{|-i|}(1)\right)\right)+a b\left(\sum_{i=0}^{s-2} \rho_{A}^{|i+1|}(1)+\sum_{i=0}^{s-2} \rho_{A}^{|-i-1|}(1)\right)\right)
\end{aligned}
$$

The proof of Remark 2 is in Appendix A8. Note that $s>3$ should hold to make equation (13) meaningful, which is a reasonable assumption to make. For $\rho_{A}(m)>0$, the sign of equation (13) depends on the term

$$
(s-2) \sum_{i=1}^{s-2}(s-i-1)\left(\rho_{A}^{|i|}(1)+\rho_{A}^{|-i|}(1)\right)-\sum_{i=1}^{s-2}\left(\rho_{A}^{|i|}(1)+\rho_{A}^{|-i|}(1)\right)
$$

Figure 1 shows the $\mathrm{MA}(1,10)$ rule autocorrelation structure under $\mathrm{AR}(1)$ asset price autocorrelation and asset price autocorrelation structure when asset price follows AR(1) process with 0.5 first order autocorrelation. 
Figure 1: Trading Strategy, MA(1,10) Rule, Autocorrelation Structure vs. Asset Price Autocorrelation Structure when the Asset Price Follows an AR(1) Process with 0.5 First Order Autocorrelation - First 30 Lags

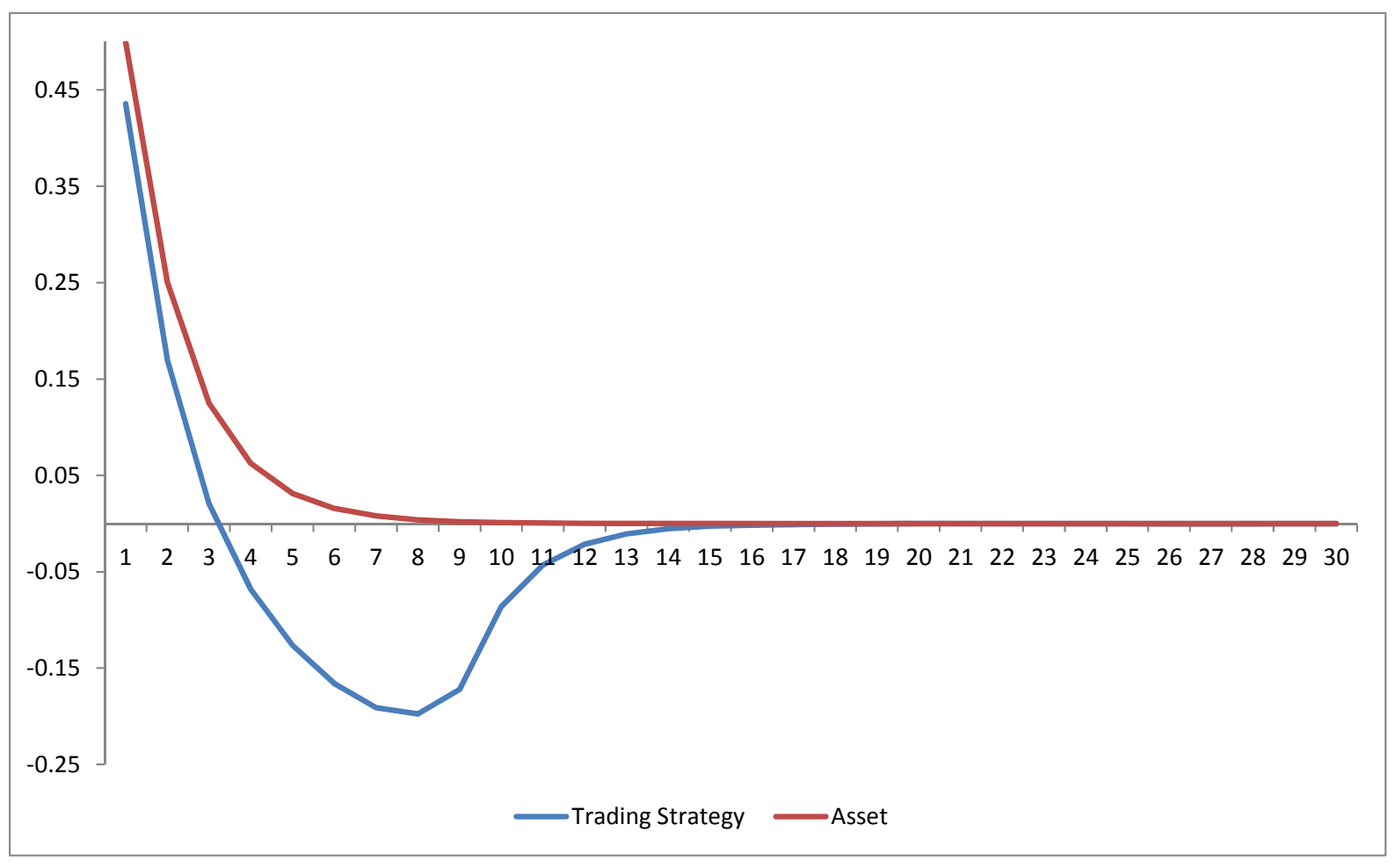

$\mathrm{x}$ - axis: lag time period, $\mathrm{m}$

y - axis: autocorrelation

Figure 1 is consistent with behavioral literatures in that the ACF of the MA rule shows initial under-reaction and delayed over-reaction or price reversals. We can see that the autocorrelation amplification of the trading strategy, compare to the underlying asset price, occurs when price reversal happens. This seemingly behavioral result is driven without assuming any behavioral aspect in determining asset price process. This might suggest that such initial under reaction and price reversal might be a product of the underlying security's autocorrelation structure and the trading rules adopted by market participants. This would be an interesting future research topic.

\subsection{The MA $(l, s)$ vs. Price Autocorrelation}

The benefit of calculating the autocorrelation functions of the $\operatorname{MA}(l, s)$ rule, $\rho_{T}(l, s, m)$, is that we can see if the imposition of the rule leads to an increase / decrease in autocorrelation in 
the money amounts associated with trading. To do this we need an overall measure of autocorrelation and a natural one is the sample statistics analogue to

Ljung-Box measure. We will use sum of squared autocorrelation and call it LB. Hence population $L B$ is computed using the population autocorrelations, $\sum_{j=1}^{\infty} \rho_{j}^{2}$ and sample $L B$ statistic is computed using the sample autocorrelations, $\sum_{j=1}^{\infty} \hat{\rho}_{j}^{2}$. For a simple model this can be calculated analytically with population values. For an $\operatorname{AR}(1)$ with correlation coefficient $\rho$, $\mathrm{P}_{\mathrm{t}}=\beta+\rho \mathrm{P}_{\mathrm{t}-1}+v_{\mathrm{t}}$ where $\mathrm{P}_{\mathrm{t}}$ is price of an asset at time $\mathrm{t}$ and $v_{\mathrm{t}}$ is the residual term from the estimated regression equation, population $L B=\rho^{2} /\left(1-\rho^{2}\right),|\rho|<1$. This section presents relative $L B$ based on price autocorrelation, which is assumed to follow an $\mathrm{AR}(1)$ process. By investigating the statistics of the $\operatorname{MAbull}(l, s)$ autocorrelation structure we can gain a good idea of how the autocorrelation structure of the $\operatorname{MAbull}(l, s)$ rule amplifies that of the price itself.

MOP investigate the correlation structure of the momentum strategy to find "Time series momentum strategies are positively correlated within an asset class, but less so than passive long strategies.” At first glance, it looks like their result is contradicting our result. However, close investigation of their trading strategy reveals that it is consistent with our result. Their strategy is stated as follows. "We consider whether the excess return over the past $k$ months is positive or negative and go long the contract if positive and short if negative, holding the position for $h$ months.” This is 'buying the past winner' strategy. The MAbull rule investigated in our paper, as previously discussed, does exactly the opposite. Although we have not explicitly shown this, extension of our result should predict that the impact of the trading strategy used in MOP would decrease the amount of autocorrelation, and this is what they find. Hence their result can be seen as a possible empirical confirmation of our theoretical result.

As previously stated, although we apply this model to the price process only, the application of the model does not need to be restricted to the price process only. It can also be applied to returns or earnings or to any process that would have time series momentum. Typical return autocorrelation lies between -0.2 and 0.2 while typical price autocorrelation, if the process is stationary, is higher than 0.6. This motivates Table 3 and 4. Table 3 reports relative population $L B$ measures for the first order autocorrelation, $\rho_{A}(1)$, from -0.2 to 0.2 and Table 4 reports for $\rho_{A}(1)$ larger than 0.6 in the increment of 0.05 . 
Table 3: Relative* Population $L B$ Measures when the Asset Price Process Follows AR(1) Process with the parameter $\rho_{A}(1)$ from -0.2 to $0.2, \rho_{A}(1)$ incrementing by 0.5 cumulated up to 100 Lags

\begin{tabular}{crrrrr}
\hline Autocorrelation & $\mathrm{MA}(1,5)$ & $\mathrm{MA}(1,10)$ & $\mathrm{MA}(1,20)$ & $\mathrm{MA}(1,50)$ & $\mathrm{MA}(1,100)$ \\
\hline-0.20 & 2.4159 & 1.5071 & 1.2131 & 1.0766 & 1.0369 \\
-0.15 & 3.7055 & 2.0310 & 1.4515 & 1.1668 & 1.0812 \\
-0.10 & 7.5538 & 3.6667 & 2.2114 & 1.4583 & 1.2250 \\
-0.05 & 29.1607 & 13.2707 & 6.7679 & 3.2275 & 2.1011 \\
0.05 & 32.4513 & 17.0475 & 9.0806 & 4.2432 & 2.6232 \\
0.10 & 9.0363 & 5.5357 & 3.3738 & 1.9720 & 1.4895 \\
0.15 & 4.5158 & 3.2538 & 2.2329 & 1.5160 & 1.2616 \\
0.20 & 2.8410 & 2.3971 & 1.8056 & 1.3455 & 1.1764 \\
\hline
\end{tabular}

* Values are normalized relative to the underlying process

Table 4: Relative* Population $L B$ Measures when the Asset Price Process Follows AR(1) Process with the parameter $\rho_{A}(1)$ from 0.6 to $0.95, \rho_{A}(1)$ incrementing by 0.5 cumulated up to 100 Lags

\begin{tabular}{crrrrr}
\hline Autocorrelation & \multicolumn{1}{c}{$\mathrm{MA}(1,5)$} & $\mathrm{MA}(1,10)$ & $\mathrm{MA}(1,20)$ & $\mathrm{MA}(1,50)$ & $\mathrm{MA}(1,100)$ \\
\hline 0.60 & 0.6554 & 1.1268 & 1.2536 & 1.1542 & 1.0859 \\
0.65 & 0.5394 & 1.0253 & 1.2250 & 1.1570 & 1.0898 \\
0.70 & 0.4432 & 0.9131 & 1.1851 & 1.1617 & 1.0959 \\
0.75 & 0.3550 & 0.7885 & 1.1244 & 1.1672 & 1.1048 \\
0.80 & 0.2736 & 0.6512 & 1.0293 & 1.1697 & 1.1173 \\
0.85 & 0.1979 & 0.5019 & 0.8831 & 1.1562 & 1.1334 \\
0.90 & 0.1275 & 0.3419 & 0.6685 & 1.0788 & 1.1428 \\
0.95 & 0.0631 & 0.1741 & 0.3743 & 0.7880 & 1.0428 \\
\hline
\end{tabular}

* Values are normalized relative to the underlying process

As $s$ increases, for $l=1$, the autocorrelation of the MA rule amplifies, see Table 3 and 4 . This result highlights the fact that the MA rule traces the price autocorrelation structure and amplifies it, although the rule does not explicitly identify the structure. Looking at Table 3 and Table 4, we see that for plausible values, the strategy has led to increased LB for 'short' rules (1 - 5 or $1-10)$ and low autocorrelation (returns) whilst it has increased autocorrelation for 'long' rules (1 - 50 or $1-100)$ and higher autocorrelation (prices). What these results indicate is that when time series (price, earnings, returns) autocorrelation is low an MA rule with a small $s$ should be used whilst an MA rule with a large $s$ should be used with a highly 
autocorrelated process. As we discussed earlier, the application of our current model is not necessarily restricted to the price process. Based on our results in this section, when the MA rule is employed with a price MA, where price autocorrelation is very high, an MA rule with a large $s$ would be appropriate. And when the MA rule is applied to a return process, which has lower autocorrelation than the price process in general, a smaller $s$ would be suitable.

\section{Empirical Evidence}

\subsection{Detrended Log Price}

The previous section has focused on analysing how the MA rule amplifies price autocorrelation structure in a simulated setting where we know the actual price autocorrelation structure. This allowed us to see situations where autocorrelation is amplified. It would also be meaningful to apply the MA rule to real historical data. In this section, we investigate the MA rule and its amplification of the autocorrelation with 15-year daily data of 11 major international stock indices from 1998 to 2012. Table 5 lists the 11 international equity indices and Table 6 presents their descriptive statistics.

Table 5: List of 11 International Equity Indices

\begin{tabular}{lc}
\hline \multicolumn{1}{c}{ Country } & Index \\
\hline US & S\&P500 Index \\
Canada & S\&P/TSX Index \\
UK & FTSE 100 Index \\
France & CAC 40 Index \\
Germany & DAX 30 Index \\
Italy & FTSE MIB Index \\
Netherlands & AEX Index \\
Japan & NIKKEI 225 Index \\
Korea & KOSPI Index \\
China & Hang Seng Index \\
Australia & S\&P/ASX 200 Index \\
\hline
\end{tabular}

Table 6: Descriptive Statistics of (Normalized relative to the first observed price of 1998) International Indices from 1998 to 2012 


\begin{tabular}{lrrrr}
\hline \multicolumn{4}{c}{ Mean } & \multicolumn{3}{c}{ Variance } & \multicolumn{2}{c}{ Skewness } & Kurtosis \\
\hline US & 1.2356 & 0.0336 & -0.3066 & 2.4448 \\
Canada & 1.4901 & 0.1393 & 0.0704 & 1.6581 \\
UK & 1.0436 & 0.0226 & -0.4853 & 2.2771 \\
France & 1.3937 & 0.1032 & 0.6425 & 2.5024 \\
Germany & 1.2873 & 0.0922 & -0.1965 & 2.2567 \\
Italy & 1.1949 & 0.1303 & 0.0384 & 2.0807 \\
Netherlan & 1.0051 & 0.0786 & 0.4811 & 2.2236 \\
Japan & 0.8366 & 0.0471 & 0.4428 & 1.9226 \\
Korea & 2.9595 & 1.7812 & 0.2356 & 1.6963 \\
China & 1.5133 & 0.2161 & 0.3180 & 2.1824 \\
Australia & 1.5622 & 0.1540 & 0.6287 & 2.6500 \\
\hline
\end{tabular}

* Sample date from January 2, 1998 to December 31, 2012

Rather than reporting the trade result over the entire sample period together, we report each year's trading separately so that it could be easier to interpret. Since we have approximately 250 trading days each year, it seems reasonable to use a 100 day look back period in estimating log price trend $(\mu)$ and long term moving average (SMA). Also 100 days is consistent with our theoretical result in Table 3 and 4 that a longer period MA rule is suitable to a time series with high first order autocorrelation. A 200 day lag is chosen to compute a sample $L B$ as discretized in Section 4.3. A lag length of 200 is the maximum number of lags that provides meaningful autocorrelations. A 240-day lagged autocorrelation in 250 trading observation can be calculated but would likely to be noisy and very close to 1 . Figure 2 depicts the linear trend line of S\&P500 log price from January 2, 2009 to May 31, 2011 as an example. Table 5 reports the detrended log price first order autocorrelation for international indices used in this section.

Figure 2: Linear Trend Line of S\&P500 Log Price 


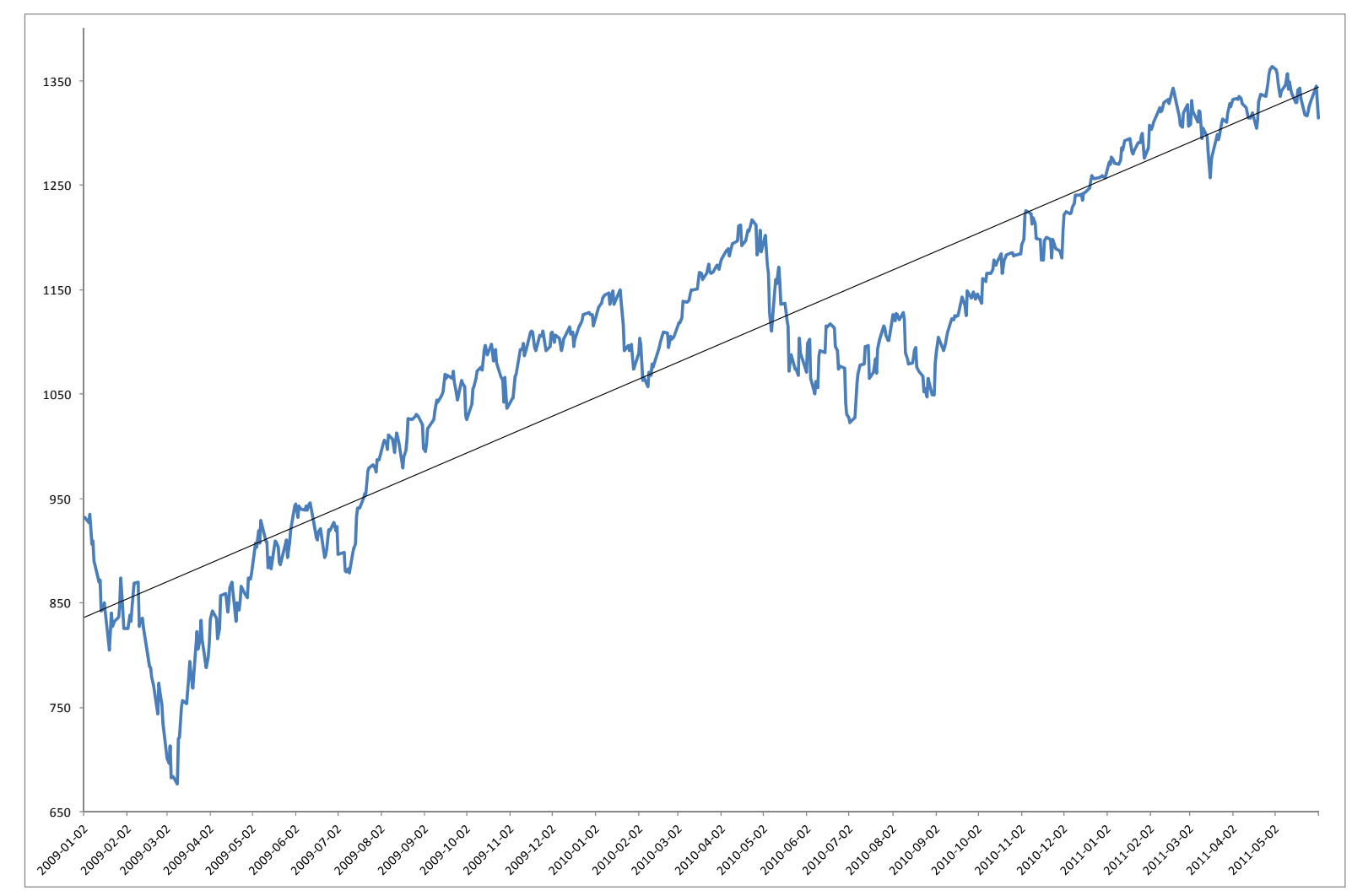

* Sample date from January 2, 2009 to May 31, 2012

In figure 2, we can see that although the index level has moved up significantly from 931.8 to 1314.55 between January 2, 2009 and May 31, 2011, the detrended log price has actually decreased. If a manager only takes advantage of upward market movement in general to generate profit, the profit is not due to the manager's ability to actively manage the portfolio. Therefore this is the reason why we use detrended price process instead.

Of course, the number of days can vary for different parameters. We uniformly apply 100 day to all the parameters to keep the empirical investigation simple. We expect the main result would not change significantly in other cases. Any activities or decisions made at time $t$ will be conditioned on the information up to time $t-1$ and hence we assume that a manager only has information up to time $t-1$ at time $t$. This means $\mu$ will be computed based on the log prices from $t-101$ to $t-1$. Then $\mu t$ is subtracted from the log price at $t$ to estimate $q(t)$. The $\mathrm{C}(t-$ 1 ) is computed based on $\mathrm{q}(t-1)$ and a $\mathrm{MA}(1,100)$ at time $t-1$, then the holding position at time $\mathrm{t}$ is decided using $\mathrm{C}(t-1)$.

The profitability of the moving average trading rule would be another interesting topic for further study. This paper is about the autocorrelation structure of the trading positions rather than the profitability of the trading rule. Deviating from the main focus and discussing 
the empirical profitability could keep the discussion away from the main theme of the paper. We try to avoid this by focusing only on the empirical autocorrelation structure of S\&P500 detrended log prices and the critical values. Hence in this empirical section, we will focus on the aggregate level of the autocorrelation in the detrended log price process, $\mathrm{q}(t)$, and the critical value, $\mathrm{C}(t)$.

\subsection{Empirical Autocorrelation Function}

Since we investigated the index level trend of international indices, an investigation of the autocorrelation structure of the major international indices should follow. Figure 6 presents the historical autocorrelation structure. To be consistent with the rest of the empirical section, we use a lag length of 200 in Figure 6.

Figure 3: Historical Daily Major International Indices Detrended Log Price Process

\section{Autocorrelation Structure}

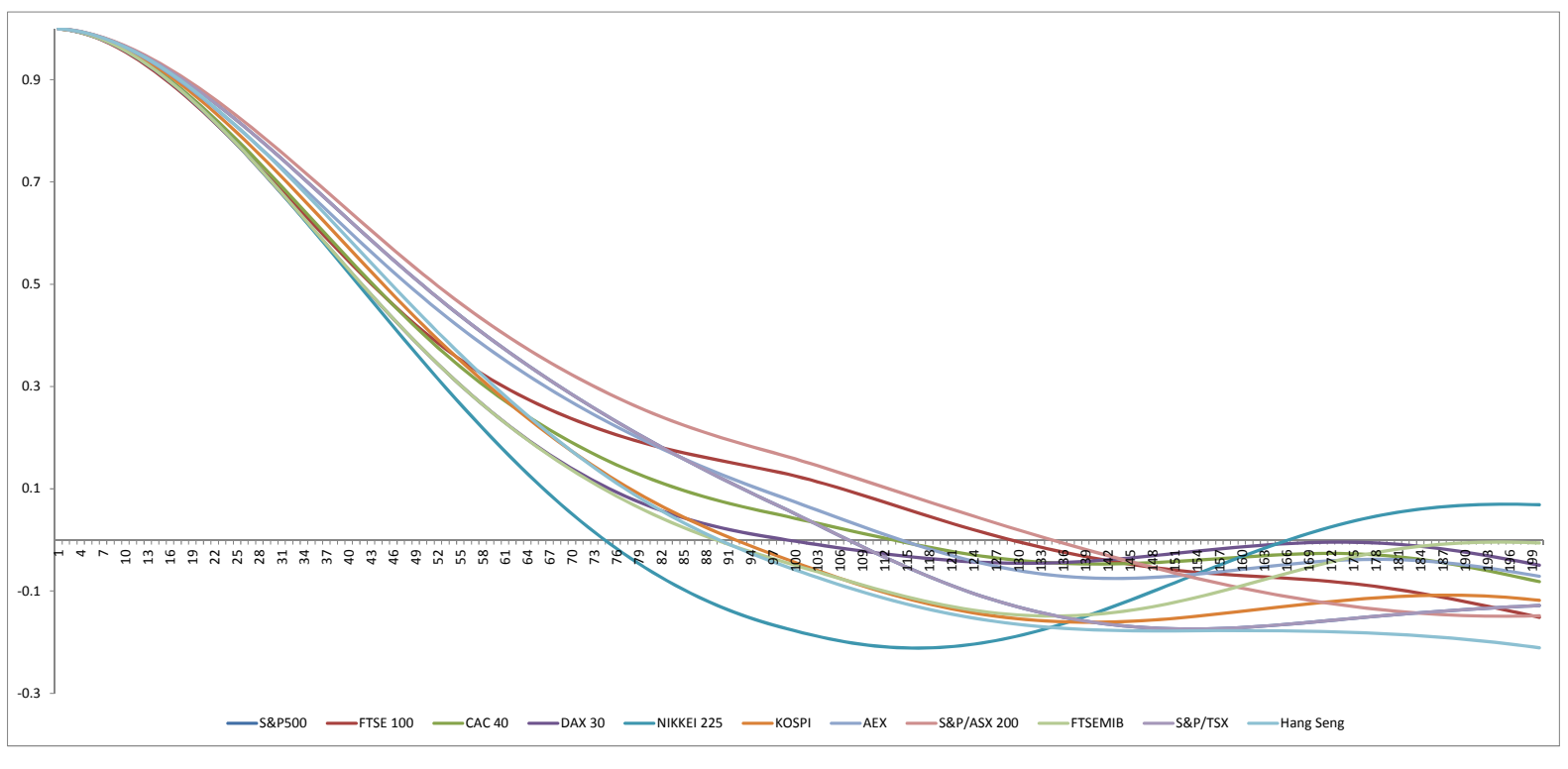

$\mathrm{x}$ - axis: lag time period, $\mathrm{m} \quad \mathrm{y}$ - axis: autocorrelation

* Sample date from January 2, 1998 to December 31, 2012

Just like the simulated result, the empirical result in Figure 3 is also consistent with behavioral literatures in that they show initial under-reaction and delayed over-reaction or price reversal. Sample $L B$ statistics are computed; they are truncated at the 200th autocorrelation and are presented in Table 8. 
Table 8: Relative sample $L B$ Statistics for Indices (Detrended Log Prices) and C $(t)$

\begin{tabular}{|c|c|c|c|c|c|c|c|c|c|c|c|}
\hline & S\&P500 & S\&P/TSX & FTSE 100 & CAC 40 & DAX 30 & FTSE MIB & AEX & NIKKEI 225 & KOSPI & Hang Seng & S\&P/ASX 200 \\
\hline 1998 & 1.245 & 1.229 & 1.060 & 0.957 & 1.093 & NA & 0.885 & 0.640 & 0.880 & 1.053 & 1.179 \\
\hline 1999 & 0.645 & 1.218 & 1.140 & 1.116 & 1.111 & 1.410 & 1.210 & 1.097 & 1.023 & 1.148 & 0.645 \\
\hline 2000 & 0.848 & 1.198 & 1.050 & 0.903 & 1.367 & 2.432 & 0.769 & 1.952 & 1.038 & 1.396 & 1.343 \\
\hline 2001 & 1.046 & 0.925 & 1.014 & 1.037 & 1.024 & 1.040 & 1.039 & 1.303 & 0.800 & 0.966 & 1.166 \\
\hline 2002 & 1.716 & 1.771 & 1.483 & 1.728 & 1.586 & 1.822 & 1.609 & 1.137 & 1.754 & 1.175 & 1.228 \\
\hline 2003 & 1.346 & 1.765 & 1.638 & 1.691 & 1.282 & 1.574 & 1.299 & 1.910 & 1.539 & 0.944 & 1.037 \\
\hline 2004 & 1.049 & 1.090 & 1.292 & 1.064 & 1.103 & 1.015 & 0.994 & 1.586 & 1.132 & 1.637 & 1.531 \\
\hline 2005 & 0.801 & 1.015 & 1.035 & 0.969 & 1.203 & 0.930 & 1.047 & 0.550 & 1.536 & 1.099 & 1.107 \\
\hline 2006 & 1.131 & 1.236 & 1.071 & 1.246 & 1.237 & 0.959 & 1.170 & 1.139 & 1.400 & 1.585 & 1.153 \\
\hline 2007 & 1.468 & 1.505 & 1.550 & 1.488 & 1.413 & 1.463 & 1.807 & 1.338 & 0.842 & 1.370 & 2.631 \\
\hline 2008 & 0.993 & 0.865 & 0.805 & 1.005 & 0.955 & 1.103 & 0.915 & 1.031 & 0.589 & 0.871 & 0.886 \\
\hline 2009 & 1.207 & 1.503 & 0.717 & 0.884 & 0.874 & 0.753 & 0.834 & 1.930 & 1.407 & 1.371 & 1.198 \\
\hline 2010 & 1.589 & 1.452 & 0.985 & 0.916 & 1.426 & 1.279 & 1.948 & 1.344 & 1.950 & 1.303 & 1.008 \\
\hline 2011 & 1.385 & 1.335 & 1.309 & 1.473 & 1.234 & 1.338 & 1.220 & 1.072 & 1.038 & 1.575 & 1.563 \\
\hline 2012 & 1.207 & 0.918 & 1.215 & 0.970 & 0.986 & 1.001 & 1.161 & 1.416 & 0.937 & 1.009 & 1.131 \\
\hline
\end{tabular}

* Sample date from January 2, 1998 to December 31, 2012

Table 9 reports mean and the variance of the relative sample $L B$ values and t-statistics

Table 9: Mean, Variance and t-statistics of Relative Sample $L B$ Statistics

\begin{tabular}{lccccc}
\hline \multicolumn{1}{c}{ Index } & S\&P500 & \multicolumn{1}{c}{ S\&P/TSX } \\
\hline Mean & 1.1786 & 1.2684 & & & \\
Variance & 0.2958 & 0.2876 & & & \\
t-statistic & 2.3376 & 2.3376 & & & \\
\hline \multicolumn{1}{c}{ Index } & FTSE 100 & CAC 40 & DAX 30 & FTSE MIB & AEX \\
\hline Mean & 1.1575 & 1.1633 & 1.1929 & 1.2942 & 1.1938 \\
Variance & 0.2603 & 0.2907 & 0.1989 & 0.4384 & 0.3484 \\
t-statistic & 2.3436 & 2.1757 & 3.7570 & 2.5994 & 2.1538 \\
\hline \multicolumn{1}{c}{ Index } & NIKKEI 225 & KOSPI & Hang Seng & S\&P/ASX 200 & \\
\hline Mean & 1.2963 & 1.1910 & 1.2335 & 1.2538 & \\
Variance & 0.4233 & 0.3874 & 0.2492 & 0.4436 & \\
t-statistic & 2.7117 & 1.9096 & 3.6281 & 2.2154 & \\
\hline
\end{tabular}

We see that the expected value of the relative sample $L B$ statistics for all the indices are larger than 1 . Our aim is to see whether the mean of the relative sample $L B$ is statistically larger than 1 or not.

$$
H_{0}: \mathrm{E}(L B)=1 \text { and } H_{1}: \mathrm{E}(L B)>1
$$

Based on Table 8, for all indices but one, KOSPI, we reject the null hypothesis of white noise at 5\% significance level. The degrees of freedoms are 14 and hence the critical value is 2.145. For all indices but KOSPI, we cannot reject the null hypothesis at 5\% level. At 10\% level, we reject the null for all indices. From the above empirical investigation, we can conclude that 
the amplification of the overall correlation in log price occurs when the MA rule is applied. We believe this can explain why the momentum trading strategy is still valid and widely used in matured markets where there has been no significant upward trend in markets.

\section{Conclusion}

This paper first presents a technical definition of momentum in order to investigate how technical analysis, especially the MA rule, becomes profitable and theoretically demonstrates that the trading rule is profitable. The theoretical examination revealed that, in addition, price autocorrelation might be another fundamental reason for profitability, and the further investigation of the autocorrelation structure of the MA rule showed that the MA rule reflects the price autocorrelation structure, without identifying it. This analysis sheds some light on why analysts use technical trading rules. There are three main findings in this paper:

1. The MA rule identifies the presence of price trend and takes advantage of it.

2. The MA rule is a simple and straightforward way of exploiting price autocorrelation without actually knowing its structure.

3. Empirically we observe that in the case of $\mathrm{MA}(1, s)$ rules, where $\mathrm{s}$ is a time period for the short position's MA, the amplification of the autocorrelation structure is more prominent for a small $s$ when the level of autocorrelation $(L B)$ is low and for a large $s$, when the level of autocorrelation $(L B)$ is high

The first finding is already well known and discussed in the earlier literature. We confirm it by constructing a simple model with a single asset. The second finding has not been investigated in the previous literature due to the lack of a precise technical definition of momentum. We show this with simulated results and empirical data from 6 international indices from developing and mature markets. The third finding suggests what length of $s$ should be applied in employing the MA rule. In particular our results indicate the different treatment for price and return based on the difference in the level of autocorrelation.

These findings should be considered important in themselves since they identify why the MA rule is used. However, the practical application of this is also interesting. One of many natural questions that can be asked is how well would the MA rule would perform if the full price autocorrelation structure was known. After all, we saw that the MA rule is all 
about simplification of conditioning on the past price information. Therefore, if we use the entire past price information, this should outperform the MA rule. However, in practice the tradeoff between the inefficiency and the cost of knowing the full autocorrelation would justify the use of the MA rule. This weakness of autocorrelation structures will be exacerbated by estimation error. Nevertheless the extent of the efficiency gain would be a worthy area for future research. 


\section{Reference}

Acar, E., Satchell, S., 1997. A theoretical analysis of trading rules: an application to the moving average case with Markovian returns. Applied Mathematical Finance 4, 165 - 180

Achelis, S., 2000. Technical Analysis from A to Z. 2nd Edition, McGraw-Hill Companies

Ahn, D. H., Conrad, J., Dittmar, R. F., 2003. Risk adjustment and trading strategies. The Review of Financial Studies 16, 459-485.

Allen, F., Karjalainen R., 1999. Earnings and price momentum. Journal of Financial Economics 51, 245-271.

Bajgrowicz, P., Sxaillet, O., 2012. Size, value, and momentum in international stock returns. Journal of Financial Economics 106, 473-491.

Barberis, N., Shleifer, A., Vishny,R., 1998. A model of investor sentiment. Journal of Financial Economics 49, 307-343.

Berk, J., Green, R .C., Naik, V., 1999.Optimal investment, growth options and security returns. Journal of Finance 54,1153-1608.

Blume, L., Easley, D., O'Hara, M. B., 1994. Market Statistics and Technical Analysis: The Role of Volume. Journal of Finance 49, 153-181.

Brock, W., Lakonishok, J., LeBaron, B., 1992. Simple Technical Trading Rules and the Stochastic Properties of Stock Returns. The Journal of Finance 47, 1731 - 1764.

Brown, S. J., Goetzmann W. N., Kumar A., 1998. The Dow Theory: William Peter Hamilton’s Track Record Reconsidered. Journal of Finance 53, 1311-1333.

Chan, K., Hameed, A., Tong, W., 2000. Profitability of Momentum Strategies in the International Equity Markets. Journal of Financial and Quantitative Analysis 35, 153-172.

Chan, K., Jegadeesh, N., Lakonishok, J., 1996. Momentum Strategies. Journal of Finance, 51, 1681-1713.

Chiarella, C., He, X. Z., Hommes, C., 2006. A dynamic analysis of moving average rules. Journal of Economic Dynamics and Control, 30(9-10), 1729-1753.

Choria, T., Shivakumar L., 2006. Using genetic algorithms to gind technical trading rules. Journal of Financial Economics 80, 627-656.

Daniel, K., Hirshleifer, D., Subrahmanyam, A., 1998. A theory of over-confidence, selfattribution, and security market under- and over-reactions. Journal of Finance 53, 1839-1885.

Fama, E.F., French, K.R., 2012. Technical trading revisited: False discoveries, persistence tests, and transaction costs. Journal of Financial Economics 105, 457-472. 
Fong, W. M., Yong, H. M., 2005. Chasing trends: recursive moving average trading rules and internet stocks. Journal of Empirical Finance 12, 43-76.

Grinblatt, M., Moskowitz, T. J., 2004. Predicting stock price movements from past returns: the role of consistency and tax-loss selling. Journal of Financial Economics 71, 541-579.

Hong, K., Satchell, S., 2012. Defining single asset price momentum in terms of a stochastic process. Theoretical Economics Letters 2(3), 274-277.

Hong, H., Stein, J., 1999. A unified theory of underreaction, momentum trading and overreaction in asset markets. Journal of Finance 54, 2143-2184.

Hou, K., Karolyi, G., Kho, B. C., 2011. What Factors Drive Global Stock Returns? The Review of Financial Studies , 24 (8), 2527-2574.

Jegadeesh N., Titman S., 2001. Profitability of momentum strategies: an evaluation of alternative explanations. Journal of Finance 56, 699-720.

Lewellen, J., Nagel, S., Shanken, J., 2010. A skeptical appraisal of asset pricing tests. Journal of Financial Economics 96 (2),175-194.

Liu, L., Zhang, L., 2008. Momentum profits, factor pricing, and macro-economic risk. Review of Financial Studies 21, 2417-2448.

Lo, A. W., Mamaysky H., Wang J., 2000. Foundations of Technical Analysis: Computational Algorithms, Statistical Inference, and Empirical Implementation. Journal of Finance 55, 1705-1765.

Marx, R., 2012. Is momentum really momentum?. Journal of Financial Economics 103, 429453.

Menkhoff, L., 2010. The use of technical analysis by fund managers: International evidence. Journal of Banking and Finance, 34, 2573-2586.

Menkhoff, L., Sarno, L., Schmeling, M., Schrimpf, A., 2012. Currency momentum strategies. Journal of Financial Economics 106, 660-684.

Moskowitz T., Ooi, Y. H., Pedersen L.H., 2012. Time Series Momentum. Journal of Financial Economics 104, 228-250.

Ng, S., Perron P., 2001. Lag length selection and the construction of unit root tests with good size and power, Econometrica, 69, 1519-1554.

Reitz, S., 2006. On the predictive content of technical analysis. The North American Journal of Economics and Finance, 17(2), 121-137.

Rouwenhorst, K. G., 1998. International Momentum Strategies, Journal of Finance, Vol. 53, No. 1, pp. 267-284. 
Sadka, R., 2006. Momentum and post-earnings-announcement drift anomalies: The role of liquidity risk. Journal of Financial Economics 80, 309-349.

Sagi, J. S., Seasholes, M. S., 2007. Firm-specific attributes and the cross-section of momentum. Journal of Financial Economics 84, 389-434.

Schwager, J. D., 1992. The New Market Wizards: Conversations With America's Top Traders. John Wiley and Sons, (pg. 224)

Johnson, T.C., 2002. Rational momentum effects. Journal of Finance 57, 585-608.

Zhu, Y., Zhou G., 2009. Technical analysis: An asset allocation perspective on the use of moving averages. Journal of Financial Economics 92(3), 519-544. 


\section{Appendix}

\section{A1 Glossary}

market wide trend: a long run market-wide trend that can be due to inflation or other economic conditions. In this paper, it is noted as $\mu$.

de-meaned price process, $\boldsymbol{q}(\boldsymbol{t})$ : price process without time dependent market wide trend, computed as $q(t)=\log P(t)-\mu t$, where $\mathrm{P}(t)$ is the asset price process.

MA, $l, s$, SMA, LMA: MA is moving average; $l$ is the time period that the MA is computed for long position; $s$ is the time period that MA is computed for short position, hence $s>l$ and they overlap. SMA is the MA computed over the period of $s$, LMA is the MA computed over the period of $l$.

$\operatorname{MA}(s, l)=\operatorname{MAbull}(s, l)$ : MA bull rule constructed with $s$ and $l$ MAs

trends: Trends, in this paper, refers to upward or downward movements in de-meaned price process for more than $s$ consecutive days.

$v(s, l ; t)$ : variable $v$ at time $t$ that is dependent on moving average time period $s$ and $l$.

$r\left(\boldsymbol{s}, \boldsymbol{l} ; \boldsymbol{t}_{\mathbf{1}}, \boldsymbol{t}_{2}\right)$ : return computed over time $t_{1}$ and $t_{2}$ that is dependent on moving average time period $s$ and $l$.

$\Omega, \Omega_{m}, \Omega^{T}, \Omega_{m}^{T}: \Omega$ is the covariance matrix of $Q(t) ; \Omega_{m}$ is the covariance matrix between $Q(t)$ and $Q(t-m) ; \Omega^{T}$ is the covariance matrix of $C(s, l ; t) ; \Omega_{m}^{T}$ is the covariance matrix between $C(s, l ; t)$ and $C(s, l ; t-m)$

$\rho_{A}(m)$ and $\rho_{T}(m): \rho_{A}(m)$ is the m-period autocorrelation of asset price and $\rho_{T}(m)$ is the autocorrelation of trading strategy critical value. 
population LB and sample LB: LB is the sum of squared autocorrelations from $1^{\text {st }}$ order to pre-specified order. ( $30^{\text {th }}$ in Section 4 and $200^{\text {th }}$ in Section 5), population LB is computed with population autocorrelations and is defined as $\sum_{j=0}^{\infty} \rho_{j}^{2}$ and sample LB is computed with population autocorrelations and is defined as $\sum_{j=0}^{\infty} \hat{\rho}_{j}^{2}$.

\section{A2 Derivation of Definition 2}

$$
\begin{aligned}
& C(t)=\exp \left(\frac{\sum_{i=1}^{l} q(t-i+1)}{l}\right) / \exp \left(\frac{\sum_{i=1}^{s} q(t-i+1)}{s}\right) \\
& =\exp \left(\frac{\sum_{i=1}^{l} q(t-i+1)}{l}-\frac{\sum_{i=1}^{s} q(t-i+1)}{s}\right) \\
& =\exp \left(\sum_{i=1}^{l}\left(\frac{1}{l}-\frac{1}{s}\right) q(t-i+1)+\sum_{i=1}^{s} \frac{q(t-i+1)}{s}\right)=\exp \left(w^{\prime} Q(t)\right)
\end{aligned}
$$

\section{A3 Derivation of Lemma 1}

$$
\begin{aligned}
& \log (P(t))-\mu t>\frac{\sum_{j=0}^{m-1}(\log (P(t-j))-\mu(t-j))}{m} \\
& \Rightarrow \log (P(t))>\frac{\sum_{j=0}^{m-1} \log (P(t-j))}{m}+\frac{\sum_{j=0}^{m-1} \mu j}{m} \\
& \Rightarrow \log (P(t))>\frac{\sum_{j=0}^{m-1} \log (P(t-j))}{m}+\frac{\mu(m-1)}{2} \\
& \Rightarrow \log (P(t))>\frac{1}{m} \log \left(\prod_{j=0}^{m-1} P(t-j)\right)+\frac{\mu(m-1)}{2} \\
& \Rightarrow P(t)>\exp \left(\frac{\mu(m-1)}{2}\right)\left(\prod_{j=0}^{m-1} P(t-j)\right)^{\frac{1}{m}}
\end{aligned}
$$


$\Rightarrow(P(t))^{\frac{m-1}{m}}>\exp \left(\frac{\mu(m-1)}{2}\right)\left(\prod_{j=0}^{m-1} P(t-j)\right)^{\frac{1}{m}}$

Therefore,

$$
P(t)>\exp \left(\frac{\mu m}{2}\right)\left(\prod_{j=1}^{m-1} P(t-j)\right)^{\frac{1}{m-1}}
$$

\section{A4 Proof of Remark 1}

Since $E[X \mid Y]=E[X]+\rho \frac{\sigma_{X}}{\sigma_{Y}}(Y-E[Y])$ and $\sigma_{X \mid Y}^{2}=\left(1-\rho^{2}\right) \sigma_{X}^{2}$, the unconditional and the conditional probability distribution of $\mathrm{C}(\mathrm{t})$ can be written as

$$
\begin{gathered}
\operatorname{prob}(C(t)>1)=\operatorname{prob}\left(w^{\prime} Q(t)>0\right)=0.5 \\
\operatorname{pdf}\left(w^{\prime} Q(t) \mid w^{\prime} Q(t-1)\right) \sim N\left(\rho(1) w^{\prime} Q(t-1),\left(1-\rho^{2}(1)\right) w^{\prime} \Omega w\right) \\
\operatorname{prob}\left(w^{\prime} Q(t)>\eta \mid \xi\right)=1-\Phi\left(\frac{\eta-\rho(1)(q(t-1)-S M A(t-1))}{\sqrt{\left(1-\rho^{2}(1)\right) w^{\prime} \Omega w}}\right)
\end{gathered}
$$

where $\xi: w^{\prime} Q(t-1)=q(t-1)-S M A(t-1)$

Since $\alpha(t)=\left\{\begin{array}{l}1 \text { if } \mathrm{C}(t)>1 \Leftrightarrow w^{\prime} Q(t)>0 \\ 0 \text { if } \mathrm{C}(t)<1 \Leftrightarrow w^{\prime} Q(t)<0\end{array}\right.$ hence $E[\alpha(t)]=\operatorname{prob}(C(t)>1)$

Unconditionally, $\operatorname{prob}(\alpha(t)=1)=0.5$

Conditionally, $\operatorname{prob}(\alpha(t)=1 \mid \xi)=1-\Phi\left(\frac{-\rho(1)(q(t-1)-S M A(t-1))}{\sqrt{\left(1-\rho^{2}(1)\right) w^{\prime} \Omega w}}\right)$ 


\section{A5 Simulation Procedure}

1. We have $q(t)=\log P(t)-\mu t-c$, assume $q(t)=\gamma+\rho_{A} q(t-1)+\varepsilon_{t}$

From GS data (May 4, 1999 - May 31, 2011), estimate c, and $\sigma_{\varepsilon}$ $c=4.182011, \sigma_{\varepsilon}=0.002687, \gamma=0.0008$ and set $\mu=0$

Let $\%$ trading cost $=0 \%$, annual risk free rate: $2 \% \rightarrow$ daily risk free rate: $0.008 \%$

2. Simulate $\varepsilon \sim N\left(0, \sigma_{\varepsilon}^{2}\right)$

3. $q(t)=\gamma+\rho_{A} q(t-1)+\varepsilon_{t}, \log P(t)=q(t)+\mu t+c, C(t)=q(t) / S M A(t)$

$S M A(t)=\exp \left((1 / s) \sum_{i=1}^{s} q(t-i+1)\right), \alpha(t)=\left\{\begin{array}{l}1 \text { if } \mathrm{C}(t)>1 \Leftrightarrow w^{\prime} Q(t)>0 \\ 0 \text { if } \mathrm{C}(t)<1 \Leftrightarrow w^{\prime} Q(t)<0\end{array}\right.$

$R_{A}(t)=\log P(t)-\log P(t-1)$

4. \% Return from the MA rule:

$$
R_{T}(t+1)=\alpha(t) R_{A}(t+1)+(1-\alpha(t))\left(r_{f}\right)-T C(t+1)
$$




\section{A6 Proof of Proposition 3}

Decompose $\Omega$ into four pieces where $\Omega_{11}$ is $l \times l, \Omega_{12}$ is $l \times(s-l), \Omega_{21}$ is $(s-l) \times l$ and $\Omega_{22}$ is $(s-l) \times(s-l)$, also decompose $w^{\prime}=\left(w_{1}{ }^{\prime} \quad w_{2}{ }^{\prime}\right)$ where $w_{1}{ }^{\prime}$ is $1 \times s$ and $w_{1}{ }^{\prime}$ is $1 \times(s-l)$

Let $a=\left(\frac{1}{l}-\frac{1}{s}\right)$ and $b=\left(\frac{1}{s}\right)$

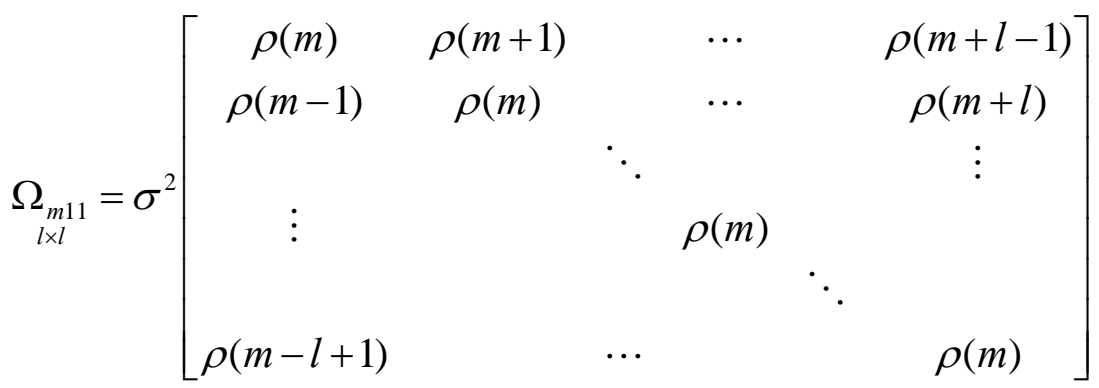

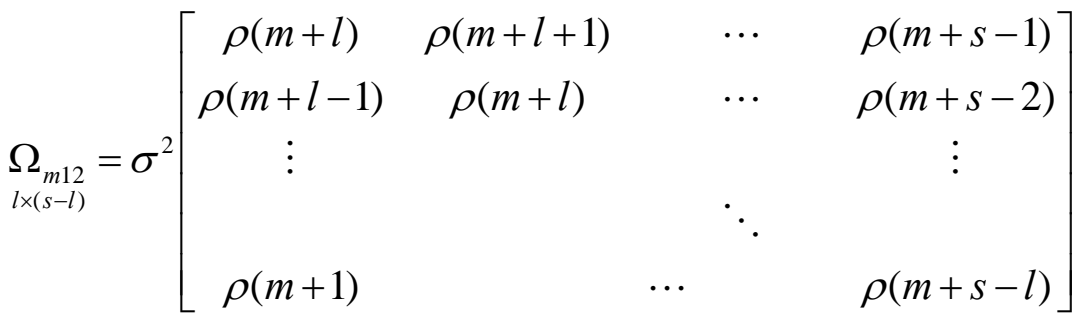

$\Omega_{\substack{m-l) \times l \\(s-l)}}\left[\begin{array}{ccccc}\rho(m-l) & \rho(m-l+1) & \cdots & & \rho(m-1) \\ \rho(m-l+1) & \rho(m-l) & & & \\ \vdots & \vdots & & \ddots & \vdots \\ \rho(m-s+1) & \rho(m-s+2) & \cdots & & \rho(m-s+l)\end{array}\right]$

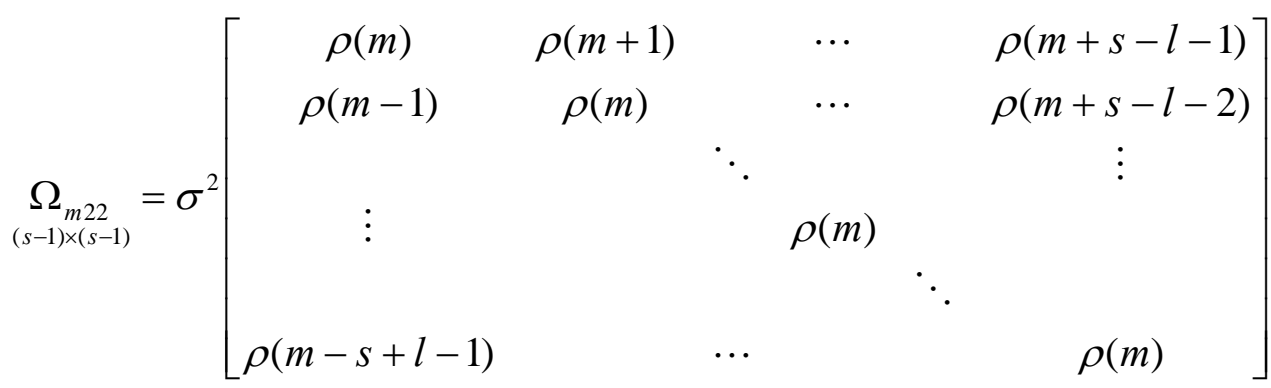


$\Omega_{m}=\left[\begin{array}{ll}\Omega_{m 11} & \Omega_{m 12} \\ \Omega_{m 21} & \Omega_{m 22}\end{array}\right], w_{1}{ }^{\prime}=\left(\begin{array}{lll}a & \cdots & a\end{array}\right)$ and $w_{2}{ }^{\prime}=\left(\begin{array}{lll}b & \cdots & b\end{array}\right)$

$$
\begin{aligned}
& w_{1}{ }^{\prime} \Omega_{m 11} w_{1}=a^{2} \sigma^{2}\left(l \rho(m)+\sum_{i=1}^{l-1}(\rho(m+i)+\rho(m-i))\right) \\
& w_{2}{ }^{\prime} \Omega_{m 22} w_{2}=b^{2} \sigma^{2}\left((s-l) \rho(m)+\sum_{i=1}^{s-l-1}(s-i-1)(\rho(m+i)+\rho(m-i))\right) \\
& w_{1}{ }^{\prime} \Omega_{m 12} w_{2}=a b \sigma^{2}\left(\sum_{j=0}^{l-1} \sum_{i=0}^{s-l-1} \rho(m+l+i-j)\right) \\
& w_{2}{ }^{\prime} \Omega_{m 21} w_{1}=a b \sigma^{2}\left(\sum_{j=0}^{l-1} \sum_{i=0}^{s-l-1} \rho(m-l-i+j)\right)
\end{aligned}
$$

$$
\left.\begin{array}{l}
w^{\prime} \Omega_{m} w=w_{1}{ }^{\prime} \Omega_{m 11} w_{1}+w_{1}{ }^{\prime} \Omega_{m 12} w_{2}+w_{2}{ }^{\prime} \Omega_{m 21} w_{1}+w_{2}{ }^{\prime} \Omega_{m 22} w_{2} \\
a^{2}\left(l \rho(m)+\sum_{i=1}^{l-1}(\rho(m+i)+\rho(m-i))\right) \\
+b^{2}\left((s-l) \rho(m)+\sum_{i=1}^{s-l-1}(s-i-1)(\rho(m+i)+\rho(m-i))\right) \\
+a b\left(\sum_{j=0}^{l-1} \sum_{i=0}^{s-l-1} \rho(m+l+i-j)+\sum_{j=0}^{l-1} \sum_{i=0}^{s-l-1} \rho(m-l-i+j)\right)
\end{array}\right)
$$




\section{A8 Calculation Check of Remark 2}

In this section, the previous result is checked with a simulation result assuming one of the most popular strategies, $1-10(l=1$ and $\mathrm{s}=10)$ and that the autocorrelation function, $\rho(m)$, follows the $\mathrm{AR}(1)$ process. 2000 sets of 5000 observations of $\mathrm{q}(\mathrm{t})$ are simulated where $q(t)=\rho q(t-1)+\varepsilon_{t}, \varepsilon \sim N(0,1)$ and $\rho=0.95$. LMA(t) and SMA(t) are computed from the simulated $\mathrm{q}(\mathrm{t})$, then subtracted to get $w^{\prime} Q(t)$. The autocorrelation of $w^{\prime} Q(t)$ is compared with (4) with $l=1$ and $\mathrm{s}=10$ and the percentage differences are reported.

Table A1: Simulation vs. Theoretical Result:

Autocorrelation of $w^{\prime} Q(t)$ when $l=1$ and $\mathbf{s}=\mathbf{1 0}$

Simulated AR(1) Theoretical AR(1) \% Difference

\begin{tabular}{llll}
\hline wOmw[1] & 0.8127 & 0.8131 & $0.0415 \%$ \\
wOmw[2] & 0.6321 & 0.6325 & $0.0629 \%$ \\
wOmw[3] & 0.4611 & 0.4619 & $0.1736 \%$ \\
wOmw[4] & 0.3039 & 0.3051 & $0.3909 \%$ \\
wOmw[5] & 0.1643 & 0.1658 & $0.9555 \%$ \\
wOmw[6] & 0.0461 & 0.0479 & $3.8566 \%$ \\
wOmw[7] & -0.0469 & -0.0448 & $-4.5953 \%$ \\
wOmw[8] & -0.1110 & -0.1083 & $-2.4332 \%$ \\
wOmw[9] & -0.1417 & -0.1387 & $-2.1642 \%$ \\
wOmw[10] & -0.1348 & -0.1317 & $-2.3007 \%$ \\
wOmw[11] & -0.1282 & -0.1251 & $-2.3803 \%$ \\
wOmw[12] & -0.1216 & -0.1189 & $-2.2386 \%$ \\
wOmw[13] & -0.1152 & -0.1129 & $-1.9212 \%$ \\
wOmw[14] & -0.1089 & -0.1073 & $-1.5143 \%$ \\
wOmw[15] & -0.1029 & -0.1019 & $-0.9676 \%$ \\
wOmw[16] & -0.0968 & -0.0968 & $0.0307 \%$ \\
wOmw[17] & -0.0910 & -0.0920 & $1.1268 \%$ \\
wOmw[18] & -0.0856 & -0.0874 & $2.0700 \%$ \\
wOmw[19] & -0.0808 & -0.0830 & $2.7619 \%$ \\
wOmw[20] & -0.0763 & -0.0789 & $3.3288 \%$ \\
wOmw[21] & -0.0727 & -0.0749 & $3.0680 \%$ \\
wOmw[22] & -0.0693 & -0.0712 & $2.6541 \%$ \\
wOmw[23] & -0.0661 & -0.0676 & $2.3749 \%$ \\
wOmw[24] & -0.0629 & -0.0642 & $2.1334 \%$ \\
wOmw[25] & -0.0600 & -0.0610 & $1.7012 \%$ \\
wOmw[26] & -0.0573 & -0.0580 & $1.1180 \%$ \\
wOmw[27] & -0.0546 & -0.0551 & $0.8748 \%$ \\
wOmw[28] & -0.0518 & -0.0523 & $0.9222 \%$ \\
wOmw[29] & -0.0491 & -0.0497 & $1.2625 \%$ \\
wOmw[30] & -0.0466 & -0.0472 & $1.3013 \%$ \\
\hline & & &
\end{tabular}

Table 3 shows that our computation is correct 